\title{
Role of Circulating Endothelial Cells and Platelet Microparticles as Markers of Angiogenesis in Chronic Myeloid Leukemia
}

Asmaanaíady', Mostafa F. Mohammed Saleh²

1 - Clinical Pthology department, Qena University Hospital, Qena, Egypt.

2. Clinical Hematology Unit, Internal Medicine department, Assiut University Hospital, Assiut, Egypt.

\begin{tabular}{l}
\multicolumn{1}{c|}{ Backiground } \\
Angiogenesis is an important component of \\
the pathogenesis of hematologic malignan- \\
cies. Circulating endothelial cells (CECS) \\
and platelet microparticles (PMPs) are pro- \\
posed as useful biosensors for angiogene- \\
sis and membrane damage in cancer.
\end{tabular}

\section{Objectives}

We investigated their possible role as markers of angiogenesis contributing to disease pathophysiology in chronic my. eloid leukemia (CML) patients and their probable value in predicting response in those treated with imatinib.

\section{Results}

The percentage of PMPs was elevated in CML patients (both groups $A \& B$ ). The mean percentaget SE of PMPS was $22.70 \pm 1.18 \%$ in controls (group C) and significantly higher in group $A(48.67 \pm 2.88 \%$; $p=0.001)$ and group $B(42.50 \pm 2.82 \% ; p=0.001)$. There was an increased number of CECs in group $A(149.33 \pm$ 23.82) vs group $B(70.96 \pm 9.58 ; p=0.009)$ and when compared to normal controls $(22.70 \pm 1.18 ; p=0.001)$. Patients with advanced phase orhigher risk disease (Intermediate and high sokal score) had slightly more PMPs and CECs than patients with chronic phase or low risk, patients on imatinib therapy who achieved CMR at 1 year showed less PMPs and CECs; however, these differenceswere not statistically significant.

\section{Patients and methods}

Peripheral blood samples were obtained from $60 \mathrm{CML}$ patients ; thirty at diagnosis (untreated) as group (A), thirty on imatinib (400 mg) therapy for at least a year as group (B) and 20 healthy controls as group (C). Flowcytometry techniques were used to quantify CECS and PMPs in all three groups. Response to therapy in group (B) was evaluated according to ELN recommendations for management of CML with complete molecular response CMR at 1 year of therapy defined as "undetectable" BCR-ABL by RQ.PCR

\section{Conclusion}

Our findings suggest the increase of PMPs and CECs number in CML patients at diagnosis could indicate possible pathogenic role as markers of angiogenessis in CML. However, their value as prognostic factors and predictors of response to therapy in CML needs larger prospective studies.

\section{Table (1)}

Circulating endothelial cells numbers and platelet microparticles percentages in CML patients on diagnosis, and after therapy and healthy controls.

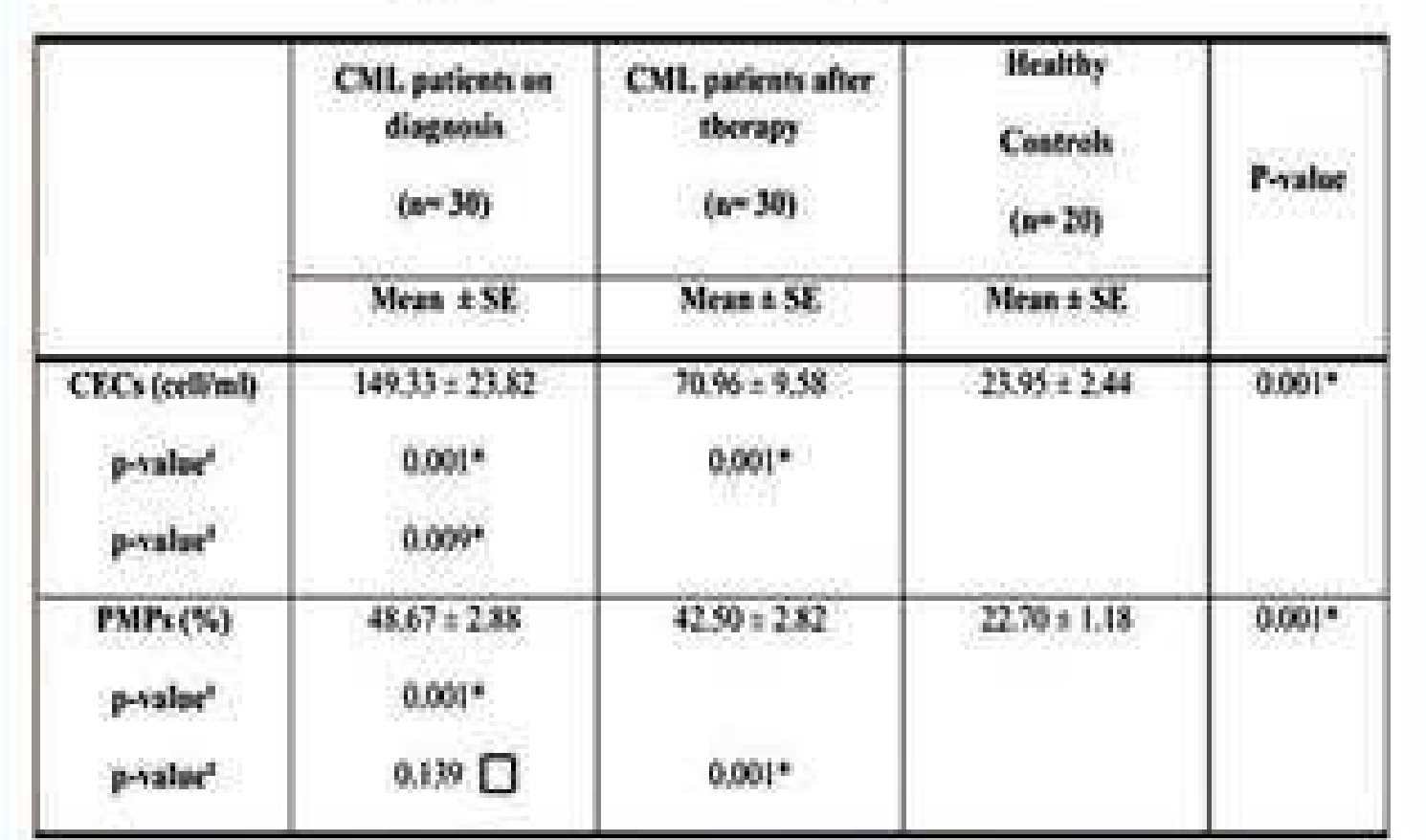

PMPs were expressed as mean \pm SE * Statistically significant difference $(P<0.05)$ and $\square$ statistically insignificant difference $(P>0.05)$. P-ralue': compared to control. P-ralue ${ }^{2}$ compared to the other group 